\title{
OPPORTUNISTIC APPROACH TO EXPLOIT WIRELESS SPECTRUM BY USE OF COGNITIVE RADIO: A REVIEW
}

\author{
Tanu Preet Singh \\ Department of Computer \\ Science \& Engineering, \\ Amritsar College of \\ Engineering \& \\ Technology, \\ Punjab Technical \\ University, Jalandhar, \\ India
}

\author{
Dr. R.K. Singh \\ $\mathrm{PhD}$, Department of \\ Electronics \& \\ Communication \\ University, Uttarakhand \\ Technical University, \\ Dehradun, India
}

\author{
Vishal Sharma \\ Department of Computer \\ Science \& Engineering, \\ Amritsar College of \\ Engineering \& \\ Technology, \\ Punjab Technical \\ University, Jalandhar,
} India

\author{
Jaspreet Kaur \\ Department of ComputerScience \& Engineering, \\ Amritsar College of Engineering \& \\ Technology, \\ Punjab Technical University, Jalandhar, \\ India
}

\begin{abstract}
Cognitive radio (CR) technology is envisaged to solve the problems in wireless networks resulting from the limited available spectrum and the inefficiency in the spectrum usage by exploiting the existing wireless spectrum opportunistically. In this paper, intrinsic properties and research on software defined cognitive radio (SDCR) are presented. Firstly brief introduction of Cognitive Radio is given along with its architecture. Then spectrum management of Cognitive Radio ad hoc networks (CRAHNs) and their main features like spectrum sensing, spectrum decision, spectrum selection and spectrum mobility are defined. At the end, Software Defined Cognitive Radio (SDCR), its hardware and software platform, along with research topics on SDCR are defined.
\end{abstract}

\section{GENERAL TERMS}

Software defined radio, Transmission detection, Receiver detection, Cognitive radio, Primary users, Cognitive radio users, Spectrum hole, Digital signal processing, ontology, signal conflict, PU detection, A2D converter, D2A converter.

\section{KEYWORDS}

SDR software defined radio, CR cognitive radio, CRAHNs cognitive radio ah hoc networks, PU primary users, QoS quality of services, SDCR software defined cognitive radio, $\mathrm{ADH}$ ad hoc networks.

\section{INTRODUCTION}

The advent of software defined radio (SDR) [1] technology offers a more sophisticated form of processing resources than prior radio technology. SDR can be defined as a mechanism in which user reconfigure radio software dynamically to fulfill the requirements of the user. Radio can be programmed to

transmit and receive on variety of frequencies. Main objective of reconfiguration is proper utilization of available bandwidth of the radio. Initially SDR technology was exclusively used for military applications but later on it was being used commercially. One technology that do not promise only processing capabilities but also provide flexible structure that is applicable to wide array of operational scenarios is Cognitive Radio (CR). An example of CR application is considering a case where vacant portions of the TV broadcast bands could be shared with unlicensed devices with sufficient intelligence to detect the licensed users and avoid causing harmful interference to those users. Unlicensed user want to access licensed bandwidth by applying various strategies and methods.

This paper describes basic definition of Cognitive Radio (CR), functionalities provided by it and its architecture that describe the working of its various components. It also describes that how available spectrum bandwidth can be monitored and selected by CR users for proper utilization of unused bandwidth. Finally this paper ends with Software Defined Cognitive Radio (SDCR) and research topics on SDCR are also defined.

\section{COGNITIVE RADIO (CR)}

CR technology is a key technology that enables cognitive radios ad hoc networks to use spectrum in dynamic manner. $\mathrm{CR}$ is a radio that can change its transmitter parameter depending upon the interaction with radio environment in which it operates. Basic characteristics of $\mathrm{CR}$ are as follows:[5,18]

\subsection{Cognitive Capability}

Cognitive capability is the capability of radio technology to sense the information from its radio environment.

\subsection{Reconfigurability}

It enables the radio to be dynamically programmed according to the radio environment to fulfill new requirements of the user. CR can be programmed to transmit and receive on a variety of frequencies.

The main objective of cognitive radio is maximum utilization of available spectrum through cognitive radio and reconfigurability as described above. Since most of the bandwidth is already assigned, main aim is to share licensed spectrum without interfering with transmission of other licensed users, the cognitive radio enable usage of temporarily unused bandwidth which is referred to as spectrum hole[5]. If this band is further needed by some licensed user then CR 
user has to move to another spectrum hole, altering its transmission power level or modulation scheme to avoid interference as shown in fig. 1

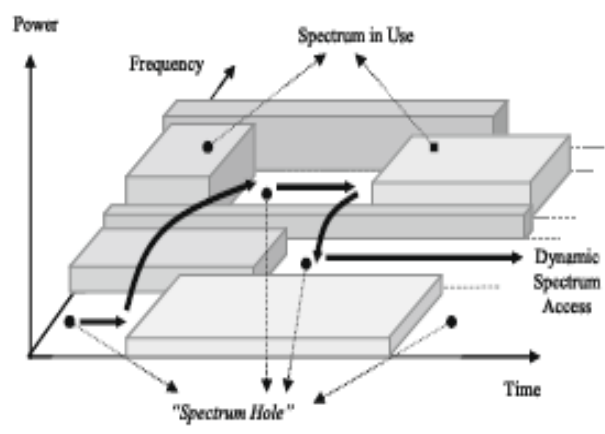

Fig: 1 Spectrum Hole concept..[3]

\section{CR ARCHITECTURE}

The architecture of CR is shown in fig. 2. at the right side of the fig. we see that SDR processing structure is accomplished in this architecture and right side of the fig. shows components involved in augmenting an SDR architecture to allow for cognitive capabilities.

At left side of the fig. PAAL layer is defined in terms of certain standard radio concepts. This is a key layer if one wants to allow for reuse of the cognitive portion of the architecture with different conventional radio implementations. That is, different radios could use different signal processing algorithms at a very low level that have no bearing on how a cognitive radio application perceives an instance of, for example, a certain kind of waveform. it also makes it possible for a CR to do things by exporting SDR primitive actions in a platform independent format.

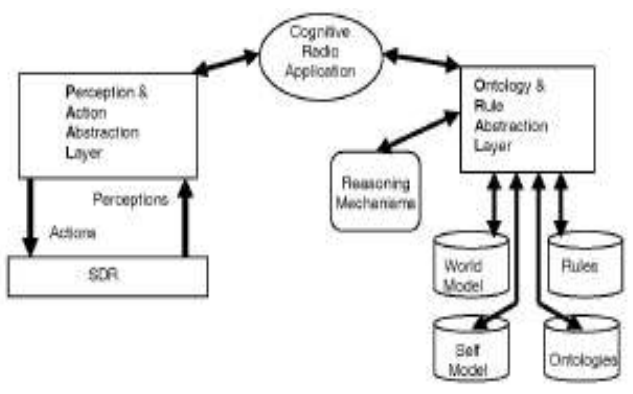

Fig : 2 CR architecture..[8]

At the right side of the figure, Rules, ontology and reasoning mechanisms components are described. Remaining components are another layer, Ontology Rule \& Abstraction Layer. It allows ontology and rule concepts to be represented in a platform-independent standard. This is important if one wants to allow the same radio implementation to be used with alternate ontology and rule reasoning platforms. Just as radio notions such as signal and waveform should have meaning independent of any particular radio implementation, so too notions such as concept, and rule should have meaning independent of any particular implementations.

\section{PROTOTYPE SIMULATION}

We have implemented a prototype simulation environment capable of handling the beacon signal conflicts. The simulation enables one or more CRs and one or more beacons to be represented in a two dimensional space. The CRs can be mobile, Means that they can be any user who can move around in simulation environment. As a radio is moved and as the various components of the environment change, an environment handler and a simulation manager ensure that the necessary events are propagated to the various elements of the simulation. Fig. 3 shows an example of the current system display and CR user interface.

A beacon signal conflict situation occurs when two beacons with opposing policies for the same channel overlap in some region. In fig. 3 the $\mathrm{CR}$ is positioned more or less equidistant from two such beacons. Such a conflict will matter to a CR only if it causes a problem with respect to a one of its goals. Suppose that the CR user has indicated a desire to use channel $\mathrm{C}$ and that a beacon signal conflict exists for C. Now how CR knows that beacon signal conflict occurs for channel C? It knows this due to series of inferences which are enabled by its ontological knowledge.

In terms of the architecture in fig. 2, SDR component process two incoming signals. Each signal is known to be associated with a certain logical channel, and certain logical channels are known to be reserved for beacons. Therefore, using its ontological knowledge, the CR concludes that the two signals it is receiving are two distinct beacon signals. Once a signal is known to be from a beacon the $\mathrm{CR}$ is able to interpret the content of the signal based on properties of the signal. So the $\mathrm{CR}$ is at that point in a position to know it is receiving a signal saying the $\mathrm{CR}$ is allowed to use channel $\mathrm{C}$ and a signal that saying the $\mathrm{CR}$ is not allowed to use channel $\mathrm{C}$. The $\mathrm{CR}$ also knows the strength of each signal. Formally, the kind of ontological reasoning just described relies upon the use of well-defined frameworks, in which definitions such as the following (schematic) definition can be encoded:

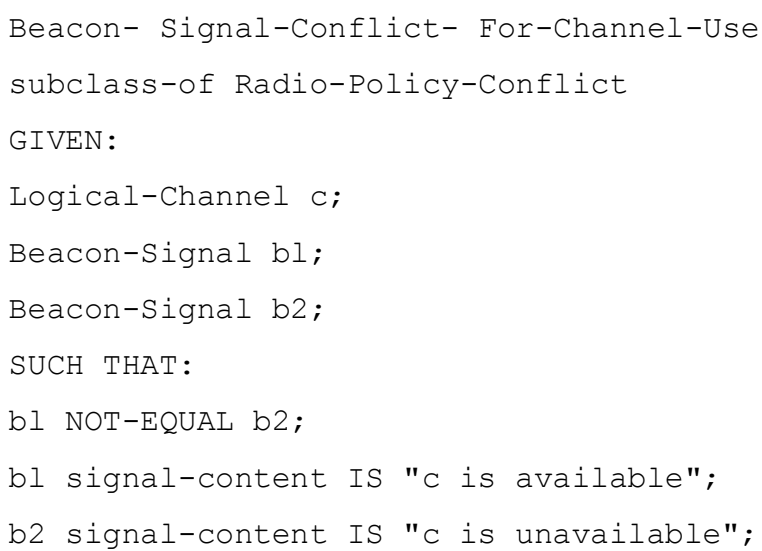

This definition provides sufficient matter for determining when a beacon signal conflict exists. 


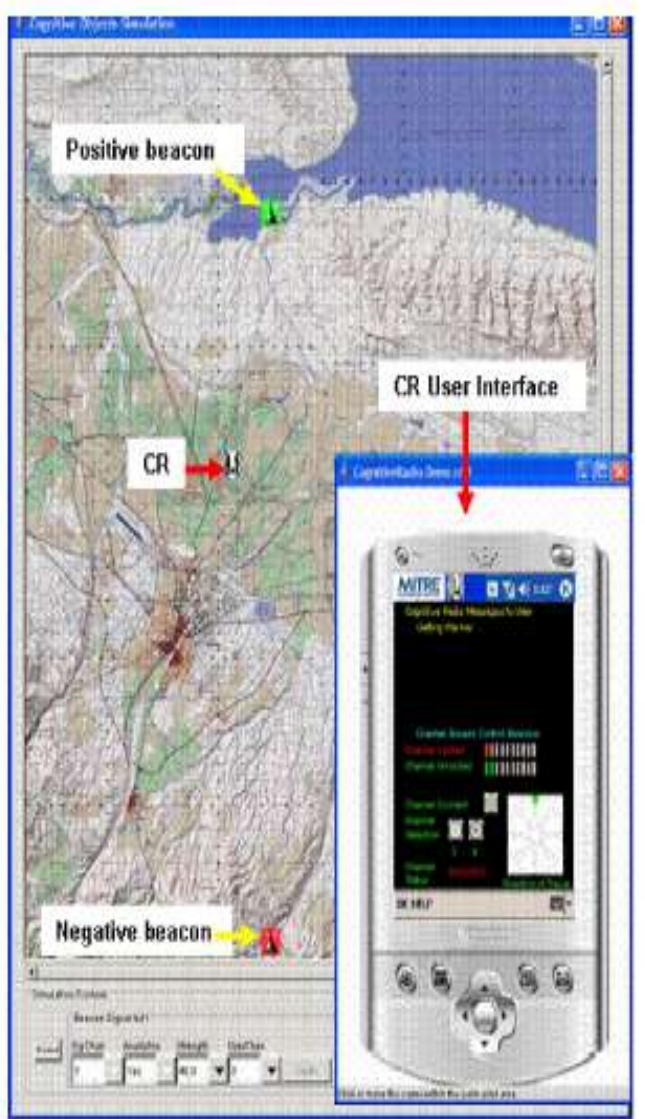

Fig: 3 Prototype screen capture and user interface..[3]

\section{SPECTRUM MANAGEMENT FRAMEWORK FOR COGNITIVE RADIO AD HOC NETWORKS}

The components of the cognitive radio ad hoc network

(CRAHN) architecture, as shown in Fig. 4a, can be classified in two groups as: primary network and the $C R$ network components.

\section{Primary network}

Primary network can be referred to existing network where primary users (PU) have license to operate in certain spectrum band. Operations of primary users (PU) are controlled through primary base station.

\section{CR network}

The CR networks are also known as secondary networks that does not have license to operate in desired band CR users are mobile so, they can communicate with each other in multi-hop manner. Hence additional functionality is required for CR users to share the licensed spectrum band. Usually, CR networks are assumed to function as stand-alone networks, which do not have direct communication channels with the primary networks. Thus, every action in CR networks depends on their local observations.

In order to adapt to dynamic spectrum environment, the CRAHN necessitate spectrum aware operations which form spectrum-cycle [5]. As shown in fig. 4b, cognitive cycle consists of following steps: spectrum sensing, spectrum decision, spectrum sharing and spectrum mobility.

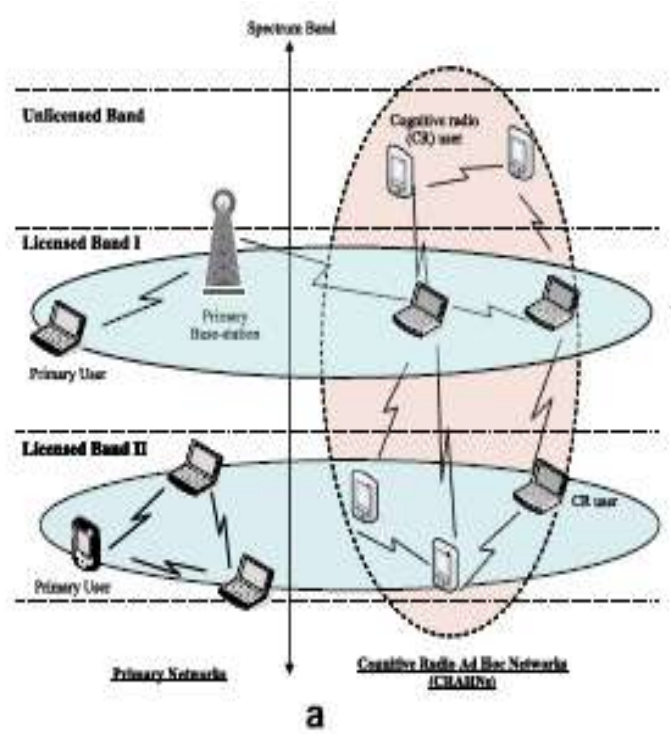

Fig: 4a CRAHN architecture..[8]

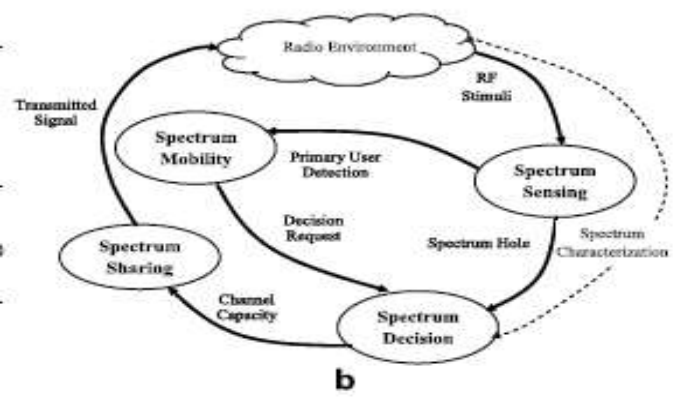

Fig: 4b Spectrum Cycle .. [3]

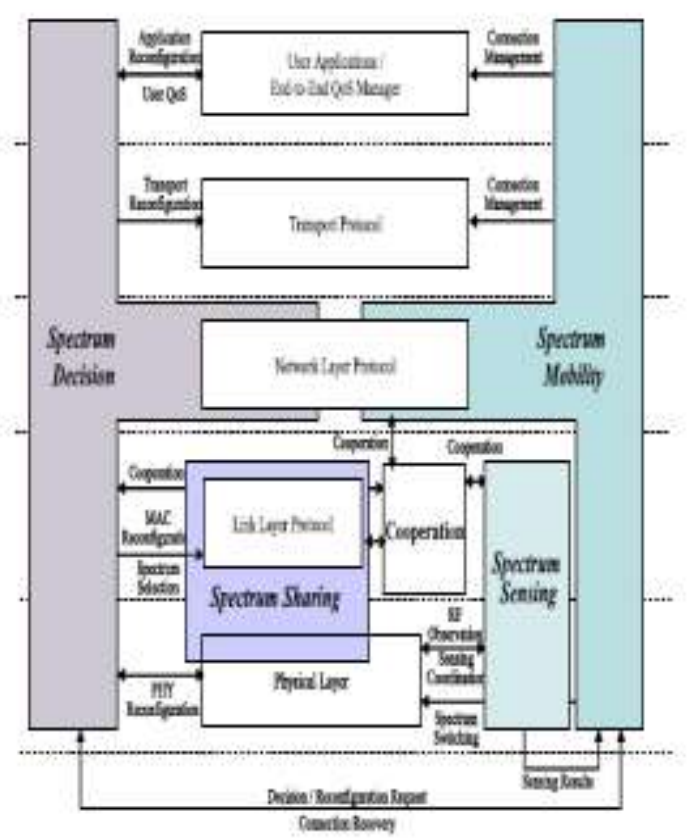


Fig: 5 Spectrum management framework .. [3]

\subsection{Features of spectrum management functions[3]}

\subsubsection{Spectrum sensing}

A CR user can be allocated to only an unused portion of the spectrum. Therefore, CR user should monitor available spectrum band and detect spectrum hole (unused space). This capability is required in following case: 1 . CR users find available spectrum holes over wide frequency range for their transmission (out-of-band sensing). 2. CR users monitor spectrum band during transmission and detect the presence of primary networks so as to avoid interference.

Spectrum sensing provides following functions as shown in fig. 6

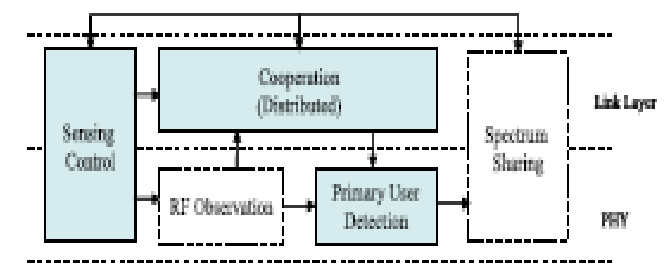

Fig: 6 Spectrum sensing structure .. [3]

4.1.1.1 PU detection- CR user observes its local radio environment. Based upon its local observations, CR users determine the presence of PU transmission and accordingly identify current spectrum availability. Generally, PU detection techniques for CRAHNs can be classified into following groups [3]: primary transmitter detector, primary receiver detector, interference temperature management.

As shown in fig. 7a, transmitter detection is based upon the detection of weak signal from primary transmitter through local observations of CR users. In transmitter detection, in order to distinguish between used and unused spectrum bands, CR users should have the capability to detect their own signal from a PU transmitter. The local FR observation used in PU detection sensing is based on the following hypothesis model [3]:

$$
r(t)= \begin{cases}n(t) & H_{0}, \\ h s(t)+n(t) & H_{1},\end{cases}
$$

Where $r(t)$ is the signal received by the CR user, $s(t)$ is the transmitted signal of the $\mathrm{PU}, \mathrm{n}(\mathrm{t})$ is a zero-mean additive white Gaussian noise (AWGN) and $\mathrm{h}$ is the amplitude gain of the channel. $\mathrm{H0}$ is a null hypothesis, which states that there is no licensed user signal in a certain spectrum band. On the other hand, $\mathrm{H} 1$ is an alternative hypothesis, which indicates that there exists some PU signal.

As shown in fig. $7 \mathrm{~b}$, the primary receiver detection aims at finding primary users that are receiving data within communication range of $\mathrm{CR}$ user. Interference temperature management accounts for the cumulative RF energy from multiple transmissions and sets a maximum cap on their aggregate level that the primary receiver could tolerate, called an interference temperature limit. As long as CR users do not exceed this limit by their transmissions, they can use this spectrum band. However, the difficulty of this model lies in accurately measuring the interference temperature since CR users cannot distinguish between actual signals from the PU and noise/interference. For these reasons, most of current research on spectrum sensing in CRAHNs has mainly focused on primary transmitter detection.

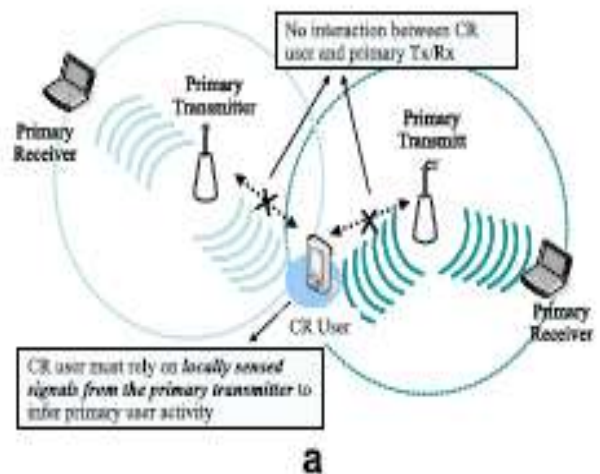

Fig: 7a Transmission detection technique ..[19]

4.1.1.2 Sensing control- This function enable CR user to perform its sensing operations adaptively to the dynamic radio environment. The main objective of spectrum sensing is to find more spectrum access opportunities without interfering with primary networks. To this end, the sensing operations of CR users are controlled and coordinated by a sensing controller which considers following main issues on: 1. how long and frequently CR users should sense the spectrum to achieve sufficient sensing accuracy in in-band sensing, and 2.how quickly CR user can find the available spectrum band in out-of-band sensing.

4.1.1.3 Cooperation- The information observed by CR users is shared with its neighbors so that sensing accuracy can be improved.

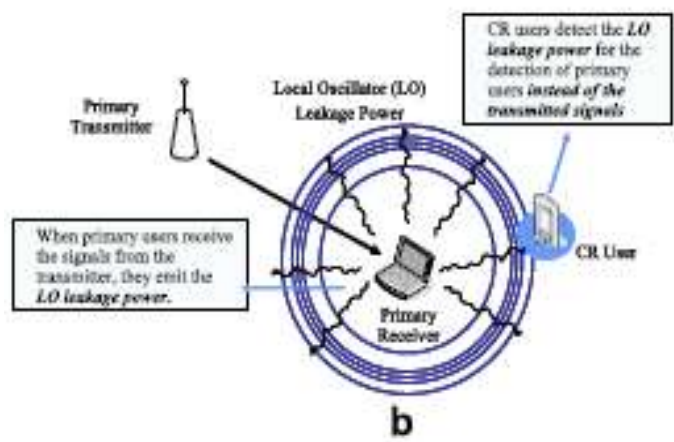

Fig: 7b Receiver Detection Technique.. [19]

\subsubsection{Spectrum Decision}

Spectrum decision is a process to decide on the best spectrum band among the available bands according to the QoS requirements of the applications Spectrum decision is closely related to the channel characteristics and the operations of PUs. Spectrum decision usually consists of two steps [19]: First, each spectrum band is characterized based on not only local observations of CR users but also statistical information of primary networks. Then, based on this characterization, the most appropriate spectrum band can be chosen. Spectrum decision needs to consider the end-to-end route consisting of multiple hops. Furthermore, available spectrum bands in CR networks differ from one hop to the other. As a result, the 
connectivity is spectrum-dependent, which makes it challenging to determine the best combination of the routing path and spectrum.

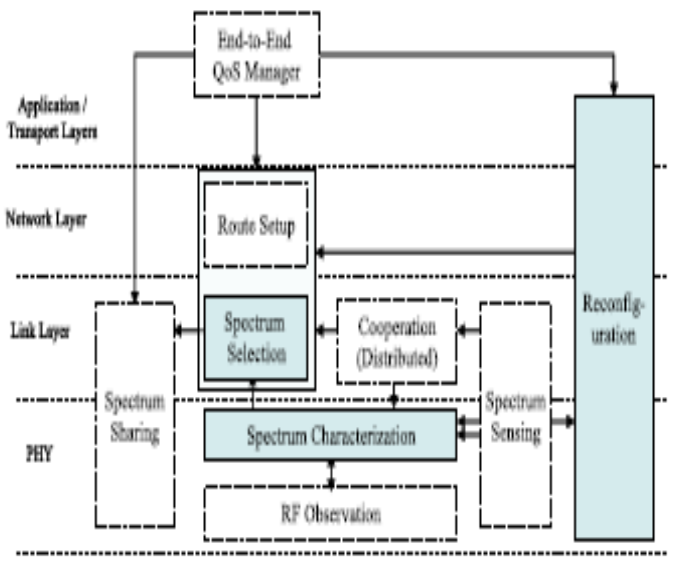

Fig: 8 Spectrum decision structure..[3]

Main functionalities required by spectrum decision are as follows:

Spectrum characterization: Based on the observation, the CR users determine not only the characteristics of each available spectrum but also its PU activity model.

Spectrum selection: The CR user finds the best spectrum band for each hop on the determined end-to-end route so as to satisfy end-to-end QoS requirements.

Reconfiguration: The CR users reconfigure communication protocol as well as communication hardware and RF front-end according to the radio environment and user QoS requirements.

$\mathrm{CR}$ ad hoc users require spectrum decision in the beginning of the transmission. CR users characterize the available spectrum bands by considering the received signal strength, interference, and the number of users currently residing in the spectrum, which are also used for resource allocation in classical ad hoc networks. However, unlike classical ad hoc networks, each CR user observes heterogeneous spectrum availability which is varying over time and space due to the PU activities. This changing nature of the spectrum usage is considered in the spectrum characterization. Based on this characterization, CR users determine the best available spectrum band to satisfy its QoS requirements. Furthermore, quality degradation of the current transmission can also initiate spectrum decision to maintain the quality of a current session.

\subsubsection{Spectrum sharing}

The shared nature of the wireless channel necessitates

Coordination of transmission attempts between CR users. So, spectrum sharing provides the capability to maintain the QoS of CR users without causing interference to the PUs by coordinating the multiple accesses of CR users as well as allocating communication resources adaptively to the changes of radio environment.

Spectrum sharing techniques are generally focused on Two types of solutions[26]: 1. spectrum sharing inside a CR network (intra-network spectrum sharing) and 2. among multiple coexisting CR networks (inter-network spectrum sharing). However, since the CRAHNs do not have any infrastructure to coordinate inter-network operations, they are required to consider the only intra-network spectrum sharing functionality. All decisions on spectrum sharing need to be made by $\mathrm{CR}$ user in distributed manner. Fig. 9 shows functional block for spectrum sharing in CRAHNs.

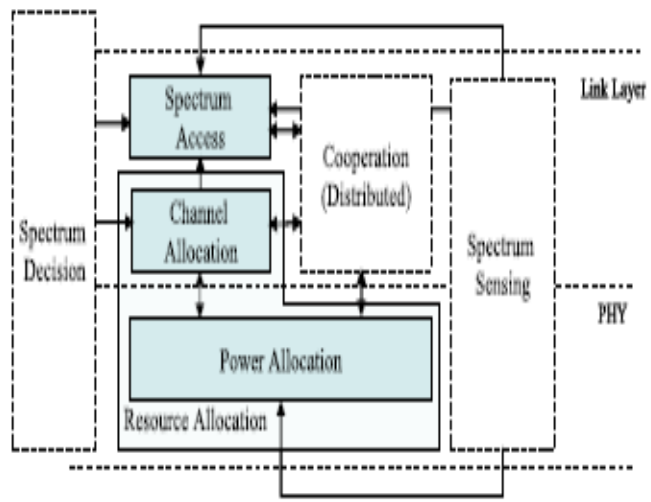

Fig: 9 Spectrum Sharing structure.. [19]

Spectrum sharing shares some features with spectrum sensing are as follows:

Resource allocation: based on QoS, CR users select the proper channel (channel allocation) and adjust their transmission power so as to achieve QoS requirements as well as resource fairness. Sensing results should not violate interference constraints.

Spectrum Access: It enables multiple CR users to share the spectrum resource by determining who will access the channel or when a user may access the channel. Once proper spectrum band is selected in spectrum decision, communication channels in that spectrum need to be assigned to a CR user while determining its transmission power to avoid interference to primary networks (resource allocation). Then CR user decides that when spectrum should be accessed to avoid collision with other users (spectrum access).

\subsubsection{Spectrum mobility}

CR users can also be known as visitors to the spectrum. Hence, if the specific portion of the spectrum is in use, is required by primary user (PU), it is needed that communication should be continued in another vacant portion of the spectrum. This notion is called spectrum mobility. With the concept of Spectrum mobility, a new type of handoff in CR networks occurs, the so-called spectrum handoff, in which, the users transfer their connections to an unused spectrum band. In CRAHNs, spectrum handoff occurs [3]: (1) when PU is detected, (2) the CR user loses its connection due to the mobility of users involved in an on-going communication, or (3) with a current spectrum band cannot provide the QoS requirements. Spectrum mobility structure for cognitive radio ad hoc networks (CRAHNs) is shown in fig. 10 . 


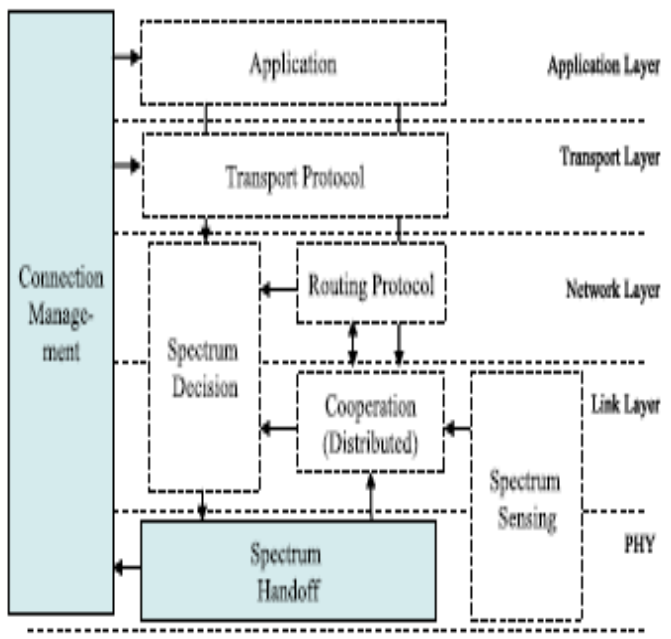

Fig: 10 Spectrum mobility structure for CRAHNs...[3]

\section{SOFTWARE DEFINED COGNITIVE RADIO}

Cognitive radio can be defined as a radio that Senses its operational (radio) environment and can dynamically adjust its radio operating parameters accordingly by collaborating wireless and wired networks. As results of sensing, when some vacant and available frequencies/time slots exist in a system, users temporally utilize the frequencies and time slots. When users would like to use several communication systems and some vacant and available frequencies and time slots that exist over some communication systems, users temporally utilize the frequencies and time slots [11]. To promote the research and development of the technology, in 2005, Ministry of Internal Affairs and Communications (MIC), JAPAN started a project regarding improved spectrum efficiency [11]. The project includes four research topics shown in Fig. 11: (1) research and development on elemental technology for cognitive radio terminals, (2) research and development on cognitive radio communication technology, (3) research and development on improved technology of frequency utilization in space domain, and (4) research and development on super-semiconductor based filter technology.

The first two research topics are closely related to the research of cognitive radio. To realize cognitive radio, there are many solutions. But SDCR is one of best technologies to control QoS (Quality of Service) of communication system, because the functions of radio communication systems are defined by software and the software is named as "Waveform." By sensing the existence of communication system by changing the waveforms, profiling the existed communication systems, and providing better communication systems to the users.

\section{RESEARCH TOPICS OF SDCR}

When cognitive radio is realized, the radio equipment must consist of hardware platform and software platform [11]. In order to realize cognitive radio hardware part, the components that are described in fig. 12 must be considered.

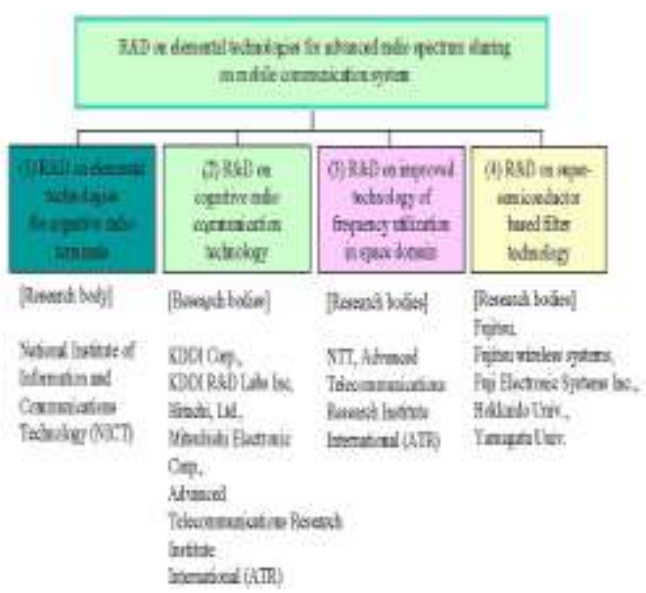

Fig: 11 Configuration of MIC project.. [11]

\subsection{Hardware platform}

The research topics are categorized into two parts: radio frequency (RF) part (shown in fig: ) and digital signal processing part (shown in fig: ). As for RF part, radio equipment must recognize the presence of several radio communication systems in the particular frequency band from VHF and UHF bands to microwave band and utilize favorite radio communication systems by the results of recognition. To realize multi-band transmission and reception, the items shown in Fig. 12 must be considered.

On the other hand, the following items must be studied for digital signal processing part.

a) Broadband, high resolution and low power

Consumption AD/DA converters.

b) Low power consumption reconfigurable digital signal processor.

In order to realize the above reconfigurable processor, four core functions must be needed: (1) Multi-IP core part, (2) Full reconfigurable part, (3) Parameter controlled reconfigurable part and (4) interfaces part, respectively.

\subsection{Software platform}

When software defined cognitive radio equipment is developed, software platform that loads waveforms to the hardware platform and profiles the existed radio communication environment must be needed as shown in Fig. 13. From this figure, there are many managers on the platform.

\section{RESEARCH CHALLENGES}

Distributed power allocation: The CRAHN users determine their transmission power in distributed manner without the support of central entity. So, interference may occur due to limitation of sensing area. So proper power control methods should be adopted to maximize the capacity with protection of transmission of PU [3]. 


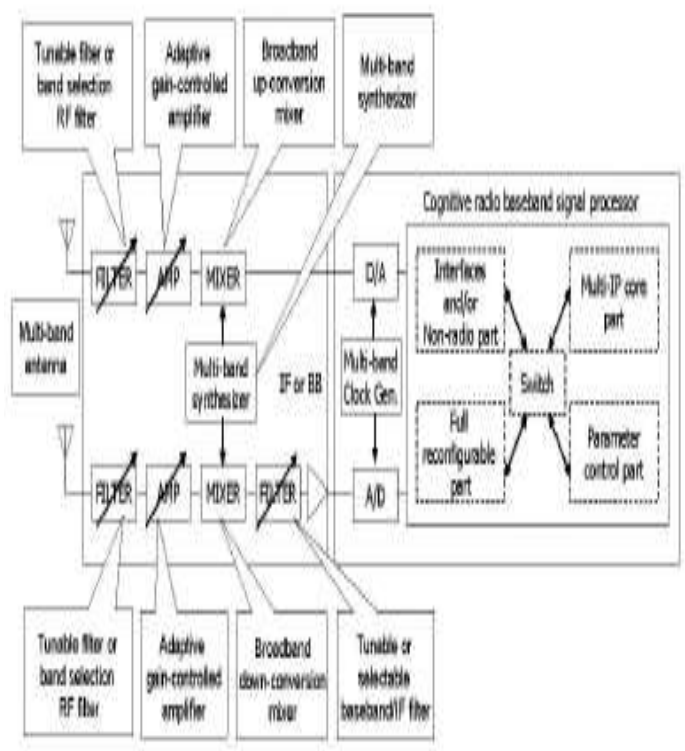

Fig: 11 Hardware platform configuration..[11]

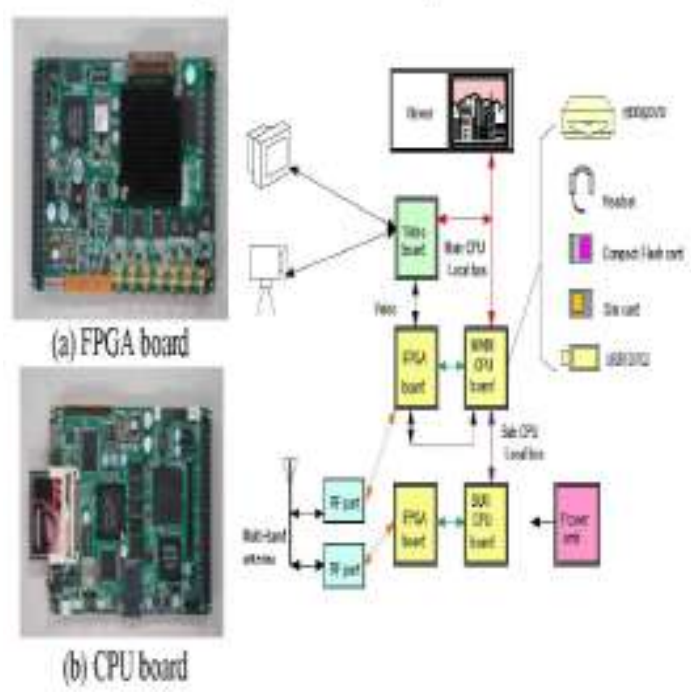

Fig: 12 Digital signal processing part..[11]

Switching delay management: The spectrum switching delay is closely related to not only hardware but also to algorithm development for spectrum sensing, spectrum decision, link layer, and routing. Thus, it is desirable to design spectrum mobility in a cross-layer approach to reduce the operational overhead among each functionalities and to achieve a faster switching time.

$A / D$ converter for sampling wideband signal: to reliably detect primary user signals through spectrum sensing, the most challenging circuits in the implementations is the $\mathrm{A} / \mathrm{D}$ converter required for sample wideband signal with large dynamic range [19].

\section{0-800MHz band TX and RX \\ $800-5200 \mathrm{MHz}$ band \\ TX and RX}
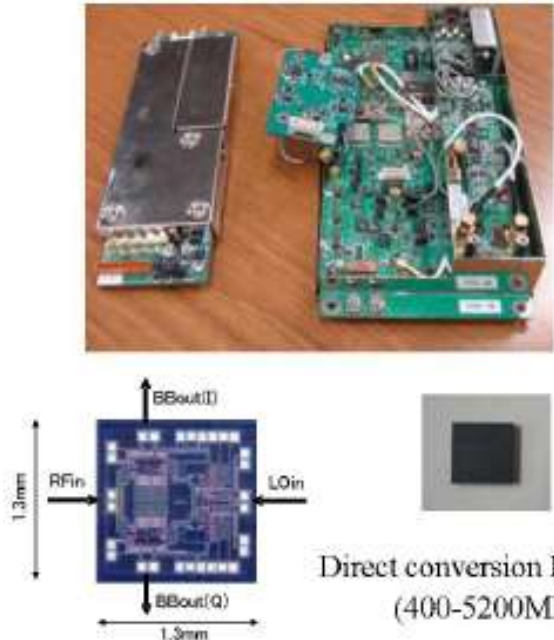

Direct conversion RX mixer $(400-5200 \mathrm{MHz})$

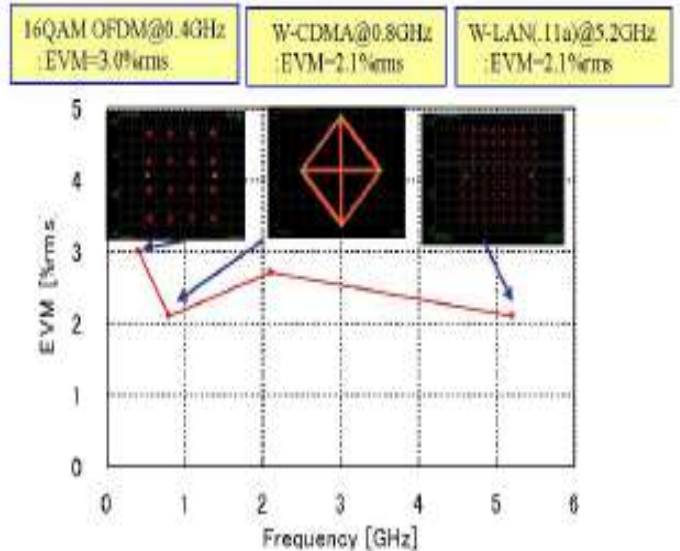

Fig: 13 RF part..[11]

Spectrum capacity estimation and different quality of services requirements necessitates new spectrum decision models.

\section{CONCLUSION}

Radio spectrum is valuable resource in wireless communication systems, and it has been the focus of many research and development efforts over trhe last several decades. Cognitive radio networks provides the key features to solve current wireless network problems by introducing opportunistic usage of the frequency band and available spectrum that are not occupied by licensed users. However they must be able to do so while minimizing the interference they cause to primary users (existing users). Hence cognitive radio must be able to sense its radio environment and dynamically adjust its radio parameters according to the requirements of the users. Many researchers are currently engaged in developing the communication technologies and protocols required for $\mathrm{CR}$ networks.

\section{REFERENCES}

[1] S. Adibi, S. Erfani, A multipath routing survey for mobile ad hoc networks, in: Proceedings of the IEEE Consumer Communications and Networking Conference (CCNC), vol. 2, January 2006, pp. 984-988. 
[2] Sahai A, Tandra R, Mishra SM, Hoven N. Fundamental design tradeoffs in cognitive radio systems. In: ACM international workshop on technology and policy for accessing spectrum; August 2006.

[3] lan F.Akyildiz, Won-Yeol, Kaushik R.Chowdhary. CRAHNs: Cognitive radio ad hoc networks. In:

[4] Raychaudhuri D, Jing X. A spectrum etiquette protocol for efficient coordination of radio devices in unlicensed bands. In: IEEE personal indoor and mobile radio communications, vol. 1; September 2003. p. 172-6.

[5] R.W. Thomas, L.A. DaSilva, A.B. MacKenzie, Cognitive networks, in: Proceedings of the IEEE DySPAN 2005, November 2005, pp. 352-

360. [6] Yucek T, Arslan H. Spectrum characterization for opportunistic cognitive radio systems. In IEEE military communications conference; October 2006. p.1-6.

[7] J.G. Proakis, Digital Communications, third ed., McGrawHill, 1995.

[8] Allen Ginsberg, Jeffrey D. Posten, and William D. Home. Toward A Cognitive Radio Architecture: Integrating Knowledge Representation With Software Defined Radio Technologies. In:

[9] Q. Zhao, L. Tong, A. Swami, Y. Chen, Decentralized cognitive mac for opportunistic spectrum access in ad hoc networks: a POMDP framework, IEEE Journal on Selected Areas in Communications (2007).

[10] P. Pawelczak, R. Venkatesha Prasad, L. Xia, I.G.M.M. Niemegeers, Cognitive radio emergency networks requirements and design, in: Proceedings of the IEEE DySPAN, November 2005, pp. 601-606.

[11] Hiroshi HARADA. Software Defined Cognitive Radio Prototype toward IMT-Advanced Wireless Communication System. In:

[13] A.M.R. Slingerland, P. Pawelczak, R.V. Prasad, A. Lo, R. Hekmat, Performance of transport control protocol over dynamic spectrum access links, in: Proceedings of the IEEE DySPAN 2007, April 2007,

pp. $486-495$

[14] W.-Y. Lee, I.F. Akyildiz, Optimal spectrum sensing framework for cognitive radio networks, IEEE Transactions on Wireless Communications 7 (10) (2008) 3845-3857.

[15] K. Akkaya, M. Younis, A survey on routing protocols for wireless sensor networks, Ad Hoc Networks (Elsevier) 3 (3) (2005) 325-349.

[16] I.F. Akyildiz, W.-Y. Lee, M.C. Vuran, M. Shantidev, NeXt generation/ dynamic spectrum access/cognitive radio wireless networks: a survey, Computer Networks Journal (Elsevier) 50 (2006) 2127-2159.

[17] D. Cabric, S.M. Mishra, R.W. Brodersen, Implementation issues in spectrum sensing for cognitive radios, in: Proceeings of the IEEE Asilomar Conference on Signals, Systems and Computers 2004,

November 2004, pp. 772-776.

[18] S. Haykin, Cognitive radio: brain-empowered wireless communications, IEEE Journal on Selected Areas in Communications 23 (2) (2005) 201-220.
[19] Lamiaa Khalid, Alagan Anpalagan. Emerging cognitive radio technology : principles, challenges and opportunities. In:

[20] L. Le, E. Hossain, OSA-MAC: a MAC protocol for opportunistic spectrum access in cognitive radio networks, in: Proceedings of the IEEE Wireless Communications and Networking Conference

(WCNC), May 2008, pp. 1426-1430.

[21] W.-Y. Lee, I.F. Akyildiz, Optimal spectrum sensing framework for cognitive radio networks, IEEE Transactions on Wireless Communications 7 (10) (2008) 3845-3857.

[22] W.-Y. Lee, I. F. Akyildiz, A spectrum decision framework for cognitive radio networks, July 2008, submitted for publication

[23] L. Ma, X. Han, C.-C. Shen, Dynamic open spectrum sharing for wireless ad hoc networks, in: Proceedings of the IEEE DySPAN, November 2005, pp. 203-213.

[24] J. Mitola III, "The Software Radio Architecture,"IEEE Commun. Mag., vol. 33, no. 5, May 1995, pp.26 38.

[25] H. Harada, "Software defined radio prototype toward Cognitive Radio Communication Systems, " IEEE Dyspan 2005, vol. 1, pp.539-547, Nov. 2005.

[26] I.F. Akyildiz, W.-Y. Lee, M.C. Vuran, M. Shantidev, NeXt generation/ dynamic spectrum access/cognitive radio wireless networks: a survey, Computer Networks Journal (Elsevier) 50 (2006) 2127-2159. 